\title{
The MAGNUM survey: positive feedback in the nuclear region of NGC 5643 suggested by MUSE ${ }^{\star}$
}

G. Cresci $^{1}$, A. Marconi ${ }^{2}$, S. Zibetti ${ }^{1}$, G. Risaliti ${ }^{1}$, S. Carniani ${ }^{2}$, F. Mannucci ${ }^{1}$, A. Gallazzi ${ }^{1}$, R. Maiolino $^{3,4}$, B. Balmaverde ${ }^{2}$, M. Brusa ${ }^{5,6}$, A. Capetti ${ }^{7}$, C. Cicone ${ }^{8}$, C. Feruglio $9,10,11$, J. Bland-Hawthorn ${ }^{12}$, T. Nagao ${ }^{13}$, E. Oliva ${ }^{1}$, M. Salvato ${ }^{14}$, E. Sani ${ }^{15}$, P. Tozzi ${ }^{1}$, T. Urrutia ${ }^{16}$, and G. Venturi ${ }^{2}$

1 INAF-Osservatorio Astrofisco di Arcetri, largo E. Fermi 5, 50127 Firenze, Italy e-mail: gcresci@arcetri.astro.it

2 Università degli Studi di Firenze, Dipartimento di Fisica e Astronomia, via G. Sansone 1, 50019 Sesto F.no, Firenze, Italy

3 Cavendish Laboratory, University of Cambridge, 19 J.J. Thomson Ave., Cambridge, UK

${ }^{4}$ Kavli Institute for Cosmology, University of Cambridge, Madingley Road, Cambridge CB2 1TN, UK

5 Dipartimento di Fisica e Astronomia, Universitá di Bologna, viale Berti-Pichat 6/2, 40127 Bologna, Italy

${ }^{6}$ INAF-Osservatorio Astronomico di Bologna, via Ranzani 1, 40127 Bologna, Italy

7 INAF-Osservatorio Astrofisico di Torino, via Osservatorio 20, 10025 Pino Torinese, Italy

8 ETH Zürich, Institute for Astronomy, Department of Physics, Wolfgang-Pauli-Strasse 27, 8093 Zürich, Switzerland

9 Scuola Normale Superiore, Piazza dei Cavalieri 7, 56126 Pisa, Italy

10 IRAM-Institut de RadioAstronomie Millimétrique, 300 rue de la Piscine, 38406 Saint Martin d'Hères, France

11 INAF-Osservatorio Astronomico di Roma, via Frascati 33, 00044 Monte Porzio Catone (RM), Italy

12 Sydney Institute for Astronomy, School of Physics, University of Sydney, NSW 2006, Australia

13 Research Center for Space and Cosmic Evolution (RCSCE), Ehime University, Bunkyo-cho 2-5, Matsuyama, Ehime 790-8577, Japan

14 Max Planck Institut für extraterrestrische Physik, Postfach 1312, 85741 Garching, Germany

15 ESO, Alonso de Cordova 3107, Casilla 19, 19001 Santiago, Chile

${ }^{16}$ Leibniz Institut für Astrophysik, An der Sternwarte 16, 14482 Potsdam, Germany

Received 22 May 2015 / Accepted 18 August 2015

ABSTRACT

\begin{abstract}
We study the ionization and kinematics of the ionized gas in the nuclear region of the barred Seyfert 2 galaxy NGC 5643 using MUSE integral field observations in the framework of the Measuring Active Galactic Nuclei Under MUSE Microscope (MAGNUM) survey. The data were used to identify regions with different ionization conditions and to map the gas density and the dust extinction. We find evidence for a double-sided ionization cone, possibly collimated by a dusty structure surrounding the nucleus. At the center of the ionization cone, outflowing ionized gas is revealed as a blueshifted, asymmetric wing of the [OIII] emission line, up to projected velocity $v_{10} \sim-450 \mathrm{~km} \mathrm{~s}^{-1}$. The outflow is also seen as a diffuse, low-luminosity radio and X-ray jet, with similar extension. The outflowing material points in the direction of two clumps characterized by prominent line emission with spectra typical of HII regions, located at the edge of the dust lane of the bar. We propose that the star formation in the clumps is due to positive feedback induced by gas compression by the nuclear outflow, providing the first candidate for outflow-induced star formation in a Seyfert-like, radio-quiet AGN. This suggests that positive feedback may be a relevant mechanism in shaping the black hole-host galaxy coevolution.
\end{abstract}

Key words. galaxies: active - galaxies: individual: NGC 5643 - ISM: jets and outflows - techniques: imaging spectroscopy

\section{Introduction}

Active galactic nuclei (AGN) have a profound influence on their host galaxies, as they are capable of ionizing large fractions of the interstellar medium (ISM) and of accelerating fast outflows. These are powerful enough both to sweep away most of the gas in the ISM and to heat the gas in the surrounding halo, inhibiting gas accretion ("negative feedback", see Fabian et al. 2012 and references therein). Fast outflows could also induce star formation ("positive feedback") in the pressure compressed molecular clouds (e.g., Silk et al. 2013) or in the outflowing gas (Ishibashi \& Fabian 2012, 2014; Zubovas \& King 2014).

Although this "positive feedback" has been invoked in recent years by several theoretical works to explain observed

* This work is based on observations made at the European Southern Observatory, Paranal, Chile (ESO program 60.A-9339). correlations between, e.g., AGN luminosities and nuclear star formation rates (Imanishi et al. 2011; Zinn et al. 2013; Zubovas et al. 2013), black hole accretion and star formation rates in AGN (Silverman et al. 2009; Mullaney et al. 2012; Silk 2013), or downsizing of both black holes and spheroids (Silk \& Norman 2009). Still very few observations of these phenomenon at work have been argued for, mostly related to spatial alignment of starforming regions or companionion galaxies with very powerful radio jets (e.g., Kramer et al. 2004; Croft et al. 2006; Elbaz et al. 2009; Feain et al. 2007; Crockett et al. 2012; Salomé, Salomé \& Combes 2015). The only known example of positive feedback at high- $z$ in a radio-quiet AGN has recently been presented by Cresci et al. 2015, who detected star-forming clumps in the host galaxy of a $z \sim 1.6$ quasar, possibly triggered by the outflow pressure at its edges, with near-IR Integral Field observations. However, we are still lacking observations of positive feedback 


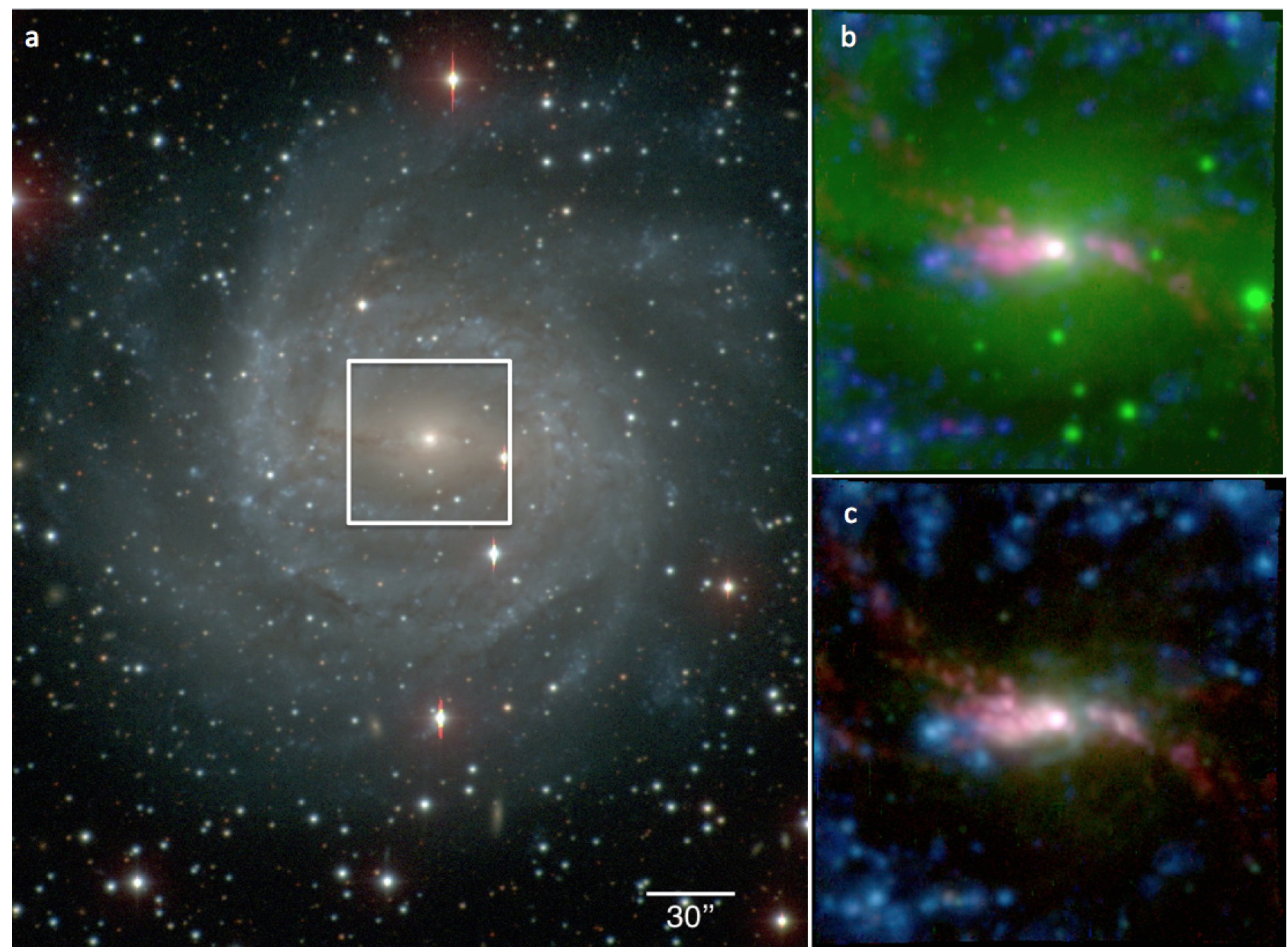

Fig. 1. Galaxy NGC 5643. Panel a) three-color (blue: $B$ band; green: $V$ band; red: $I$ band) image of the galaxy obtained with the $2.5 \mathrm{~m}$ du Pont Telescope for the Carnegie-Irvine Galaxy Survey (CGS, Ho et al. 2011). The MUSE field of view is shown with a white square. North is up and east is left. Panel b) three colors MUSE map, showing $\mathrm{H} \alpha$ emission in blue, [OIII] $\lambda 5007$ emission in red, and the integrated continuum emission from 5200 to $6000 \AA$ in green. The tip of the dust lane in the bar, visible on larger scale in Panel a, is evident next to the blue clump east of the nucleus as a gap in the continuum emission. Panel c) three-color MUSE maps, showing $\mathrm{H} \alpha$ in blue and [OIII] 25007 in red as before, and $[\mathrm{NII}] \lambda 6584$ emission in green. The two-sided AGN ionization cone traced by the filamentary [OIII] emission is prominent, as well as the strongly $\mathrm{H} \alpha$ emitting clumps between the two eastern lobes of the cone. North is up and east is left.

at lower AGN luminosity and outflow energy, which would contribute to the understanding of its importance in normal AGNs.

In this paper we present observations of the nuclear region of NGC 5643, a barred, radio-quiet Seyfert 2 galaxy at a distance of $\sim 17.3 \mathrm{Mpc}$ seen almost face on $(i \sim-27 \pm 5 \mathrm{deg}$, de Vaucouleurs et al. 1976), with a well-known ionization cone extending eastward of the nucleus, parallel to the bar (Schmitt et al. 1994; Simpson et al. 1997; Fischer et al. 2013). A three-color image ( $B, V, I$ bands) from the Carnegie-Irvine Galaxy Survey (CGS, Ho et al. 2011) is shown in panel $a$ of Fig. 1. As usually observed in barred spiral galaxies, sharp, straight dust lanes extend from the sides of the central nucleus out to the end of the bar, roughly parallel to its major axis and is more clearly visible to the east of the nucleus. The galaxy is also known to host a diffuse, low-luminosity radio jet on both sides of the nucleus, which is nearly $30^{\prime \prime}$ long (2.5 kpc, Leipski et al. 2006; Morris et al. 1985).

This galaxy was observed with the Multi Unit Spectroscopic Explorer (MUSE), the optical large-field, integral-field spectrometer at the Very Large Telescope (VLT; Bacon et al. 2010), as part of the science verification run in the framework of the Measuring Active Galactic Nuclei Under MUSE Microscope (MAGNUM) survey. This program aimed at the observation of nearby AGNs to study the physical conditions of the narrow line regions (NLRs), the interplay between nuclear activity and star formation, and the effects and acceleration mechanisms of outflows.

In the following, we adopt the systemic velocity measured by Koribalski et al. 2004, $1199 \mathrm{~km} \mathrm{~s}^{-1}$. We use $H_{0}=69.6 \mathrm{~km} \mathrm{~s}^{-1} \mathrm{Mpc}^{-1}$ and $\Omega_{M}=0.286$ (Hinshaw et al. 2013). At the distance of the galaxy of $17.3 \mathrm{Mpc}$, the angular scale is $1^{\prime \prime}=83 \mathrm{pc}$.

\section{Observations, data reduction, and analysis}

NGC 5643 was observed with MUSE during the science verification run on June 24, 2014, under program 60.A-9339 (PI Marconi/Hawthorn/Salvato). The average seeing during the 
observations, derived directly from foreground stars in the final datacube, was $F W H M=0.88^{\prime \prime} \pm 0.02^{\prime \prime}$. The nuclear region of the galaxy was observed with four dithered pointings of $500 \mathrm{~s}$ each, with the sky sampled with four shorter $100 \mathrm{~s}$ exposures in between. The data reduction was performed using recipes from the early-release MUSE pipeline (version 0.18.1, Weilbacher et al. 2014), as well as a collection of custom IDL codes developed to improve the sky subtraction, response curve, and flux calibration of the data. Further details on the data reduction will be provided in a forthcoming paper (Carniani et al., in prep.). The final datacube consists of $315 \times 315$ spaxels, for a total of almost 100000 spectra with a spatial sampling of $0.2^{\prime \prime} \times 0.2^{\prime \prime}$ and a spectral resolution from 1750 at $465 \mathrm{~nm}$ to 3750 at $930 \mathrm{~nm}$.

Moreover, NGC 5643 was observed in the X-rays by the Chandra Observatory on December 26, 2004, for $7.5 \mathrm{ks}$ with the Advanced CCD Imaging Spectrometer (ACIS; Garmire et al. 2003). Data were reduced with the Chandra Interactive Analysis of Observations 4.5 (CIAO; Fruscione et al. 2006) and the Chandra Calibration Data Base 4.6.5, adopting standard procedures. The imaging analysis was performed by applying a factor of 2 subpixel event repositioning to increase the spatial resolution (e.g., Wang et al. 2011). We therefore used a pixel size of $0.246^{\prime \prime}$, instead of the native $0.495^{\prime \prime}$.

\subsection{Modeling the stellar continuum and emission lines}

In the presence of young- or intermediate-age stellar populations, which present intense Balmer absorption (e.g., $\mathrm{EW}(\mathrm{H} \beta)$ can reach up to $8 \AA$ ), a careful subtraction of the underlying stellar continuum is important to recover the correct emission line fluxes and profiles. Although the optimal approach is to make a simultaneous fit of the stellar continuum and of the emission lines (see, e.g., Sarzi et al. 2006), with $10^{5}$ spaxels and a spectral sampling of $1.25 \AA$ this becomes computationally infeasible for a MUSE datacube on a standard desktop computer. On the other hand, considering the intensity of the emission lines we aim to study here (see next section), we can obtain sufficient accuracy by fitting the stellar continuum while avoiding the regions interested by emission lines and then extrapolating the fit over those regions. We use the pPXF code (Cappellari \& Emsellem 2004) to perform this task over the wavelength range of interest, i.e., shortward of $7000 \AA$. A linear non-negative combination of stellar spectral templates is fitted to the data via adjusting the systemic velocity and the velocity dispersion together with the coefficients of the linear combination. We consider 40 stellar templates from the simple stellar populations provided by Bruzual \& Charlot 2003, distributed in a grid of ten ages $(5.2,25,100,290,640,900 \mathrm{Myr}, 1.4,2.5$, 5.0, and $11 \mathrm{Gyr})$ and four metallicities $(0.2,0.4,1$, and 2.5 times solar). Each spectral template is converted from wavelength to velocity space as is each observed spectrum. The pPXF iteratively adjusts the systemic velocity and the Gaussian velocity dispersion of each template (this is accomplished with plain convolution in the velocity space with a Gaussian kernel; velocity and dispersion are assumed to be common to all component templates) and computes the coefficients of the linear combination of templates that best reproduce the observed spectrum. The procedure is iterated until the minimum $\chi^{2}$ and the bestfitting systemic velocity, velocity dispersion, and coefficients of the template linear combination are obtained (see Cappellari \& Emsellem 2004, for more details). The template distribution in age and metallicity allows us to cover virtually any combination of stellar populations inside a galaxy, including the very young ages that characterize the star-froming blobs. We mask regions corresponding to $\pm 750 \mathrm{~km} \mathrm{~s}^{-1}$ around the main astrophysical emission lines and around the $\mathrm{NaI}$ absorption at rest and in the galaxy's rest frame to avoid contamination from the interstellar absorption. The fit is expected to be definitely degenerate in terms of the possible linear combinations of SSPs, but this is irrelevant to our goal: a realistic model of the star formation history and metallicity distribution is not required to ensure just an accurate stellar continuum subtraction. We verify that we obtain an accurate subtraction by comparing the residuals from the fit with the estimated noise level and finding them consistent with each other.

This fitting procedure only produces reliable results if a sufficient signal-to-noise ratio $(\mathrm{S} / \mathrm{N})$ in the continuum is provided, namely $>10$ per $\AA$. In the original datacube at full resolution this is only achieved in the brightest parts; for the fainter regions, we apply an adaptive smoothing procedure, which extends the ADAPTSMOOTH code (Zibetti 2009a, Zibetti et al. 2009b) to work with spectral datacubes (AZMOOTH3, Zibetti et al., in prep.). At each spaxel, the $\mathrm{S} / \mathrm{N}$ is estimated in a narrow wavelength window on a featureless continuum of between 6000 and $6050 \AA$. If the $\mathrm{S} / \mathrm{N}$ is lower than the threshold of 20 per spectral pixel, concentric annuli of spaxels are coadded iteratively until the $\mathrm{S} / \mathrm{N}$ of 20 is reached. No more than ten annuli are coadded at each position. The smoothed cube warrants sufficient $\mathrm{S} / \mathrm{N}$ at all spaxels while preserving the effective spatial resolution as much as possible. The typical smoothing radius (in pixels, 1 pix $=0.2^{\prime \prime}$ ) varies from 1 (i.e., no smoothing) within $5^{\prime \prime}$ from the center to 2,3 , and 5 at distances of $\sim 13^{\prime \prime}, 18^{\prime \prime}$, and $25^{\prime \prime}$, respectively. Larger smoothing radii are only required in sparse regions, typically outside $25^{\prime \prime}$. Indeed, most of the analysis presented in this work is unaffected or marginally affected by the smoothing: the EELR are in fact mostly contained in the no-smoothing zone or in the two-pixel radius smoothing zone. In any case, the smoothing affects scales typically $<2$ " in diameter, hence, smaller than those we investigate in the following. The output is then processed with pPXF, as explained above, to produce a pure stellar continuum cube, stellar velocity, and stellar velocity dispersion maps.

\subsection{Emission line fitting, density, and extinction maps}

The obtained stellar continuum is subtracted from the original data, and the resulting datacube is used for the following analysis. We fit the main emission lines accessible in the MUSE wavelength range, i.e., $\mathrm{H} \beta$, [OIII] $\lambda 4959$, [OIII] $\lambda 5007$, [OI] $\lambda 6300$, [NII] $\lambda 6548, \mathrm{H} \alpha,[\mathrm{NII}] \lambda 6584,[\mathrm{SII}] \lambda 6716,[\mathrm{SII}] \lambda 6730$. To better reproduce the observed spectral profiles in the data, we use both a two Gaussian components fit and a single Gaussian fit to each line. The flux obtained with the double Gaussian is used in the following only for the spaxels where the second component has a $S / N>3$ in the fit, i.e., in the nuclear region where the line profile is more complex (see Fig. 2). This choice allows us to use the more degenerate two-components fit only where it is really needed.

From these fluxes we constructed emission line maps in the $\mathrm{H} \alpha$ [OIII] and [NII] transitions (see Fig. 1b,c). These maps already clearly indicate that the line-emitting gas presents an elongated morphology, aligned with the bar in the E-W direction. In the following, we refer to this region as the extended emission line region (EELR). As reported by Schmitt et al. 1994, the EELR is elongated both to the $\mathrm{E}$ and to the $\mathrm{W}$ of the nucleus, although it is much fainter to the $\mathrm{W}$, especially at shorter 

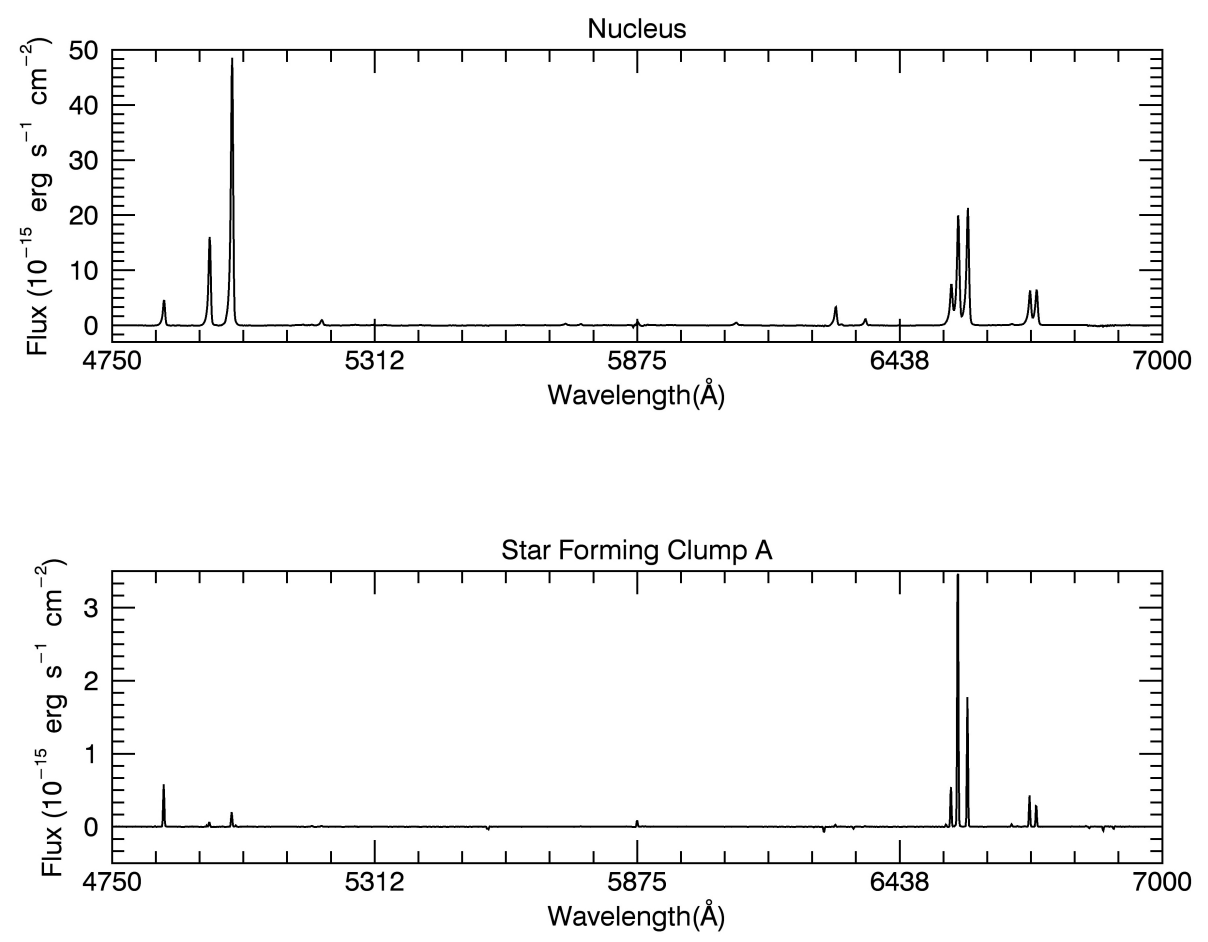

Fig. 2. Continuum subtracted, rest-frame spectra extracted from a region of $1.6^{\prime \prime} \times 1.6^{\prime \prime}$ around the nucleus (upper panel) and around the star-forming clump A (lower panel). The different excitation between the two regions is evident from the different line ratios, where [OIII] and [NII] are brighter in the nucleus, while stronger $\mathrm{H} \alpha$ and $\mathrm{H} \beta$ emission is detected in the star-forming clumps. wavelengths (e.g., Fig. 1, panels, b and c). As we discuss later, this is because of the higher extinction to the $\mathrm{W}$, indicating that the EELR is tilted with respect to the galaxy plane, and it is lying behind the disk of the galaxy to the $\mathrm{W}$, while it is seen mostly above the disk to the E.

An [OIII] emitting structure is very prominent, shown in red in Fig. 1, originating at the AGN location in the galaxy center and extending in the E-W projected directions, part of an ionization bicone (see Fig. 1, panels b and c; see also Fischer et al. 2013). A $V$-shaped ionization cone in the core of NGC 5643 was already reported by Simpson et al. (1997) using HST narrowband imaging, although the western part of the bicone was not detected in their data, probably due to lower sensitivity.

Strikingly, a blue, $\mathrm{H} \alpha$ bright region is located exactly at the tip of the eastern part of the EELR at a distance of $\sim 15^{\prime \prime}=1.2 \mathrm{kpc}$ from the nucleus, at the location where the line-emitting region reaches the dust lane of the bar (clump A, see panel $\mathrm{b}$, where the dust in the bar is visible as a dip in the green continuum), and another H $\alpha$ bright clump is located $\sim 8^{\prime \prime}$ to the southwest of clump A (clump B, see also Figs. 3 and 7). A comparison between the line ratios measured on clump $A$ and the line ratio in the nuclear region shows that the excitation mechanisms are different, with the nucleus showing an AGNlike ionization as expected, while clump A displays the typical spectrum of a star-forming region (see Fig. 2). We discuss these star-forming regions further in the following.

We estimate the electron density from the ratio between the [SII] $\lambda 6716$ and [SII] $\lambda 6730$ lines, which is a widely used indicator of this quantity (e.g., Osterbrock \& Ferland 2006). We compute the line ratio in each spaxel, and convert it to an electron density using the IRAF task temden, assuming a temperature of $10^{4} \mathrm{~K}$. The resulting electron density map is shown in Fig. 3, a, for the regions where the [SII] lines are detected with $S / N>5$. The density is higher in the nucleus, and it decreases outward by almost three order of magnitudes to a level of $\sim 10 \mathrm{~cm}^{-3}$. The density is again higher $\left(\sim 100 \mathrm{~cm}^{-3}\right)$ in the blue, $\mathrm{H} \alpha$ bright clump. We note that the density derived with this method on the nucleus is higher than the critical density for [SII] $\left(\sim 3 \times 10^{3} \mathrm{~cm}^{-3}\right)$ above which the lines become collisionally de-excited (see Osterbrock \& Ferland 2006). Therefore, the density derived in the nucleus with this method is very uncertain.

We also use the Balmer decrement $\mathrm{H} \alpha / \mathrm{H} \beta$ to derive the dust extinction map, assuming a Calzetti et al. 2000 attenuation law and a fixed temperature of $10^{4} \mathrm{~K}$. Although there could be in principle a large temperature range across the field of view, the expected variation in $A_{V}$ is \pm 0.2 for a reasonable temperature range between $5000 \mathrm{~K}$ to $20000 \mathrm{~K}$, typical of the conditions in HII regions and in the narrow- and broad-line regions of AGNs (Osterbrock \& Ferland 2006). The expected variation for a temperature of $5000 \mathrm{~K}$ or $20000 \mathrm{~K}$ is \pm 0.2 in $A_{V}$, which does not change our qualitative picture as the difference between the dusty structure W of the nucleus and the EELR to the E is one order of magnitude greater. The resulting map is shown in Fig. 3b. We find that the reddening is generally higher to the $\mathrm{W}$ of the nucleus. This confirms that the bright line-emitting gas is probably equally extended to the $\mathrm{W}$ and $\mathrm{E}$ of the nucleus, but while the $\mathrm{E}$ side is seen in front of the bar, the $\mathrm{W}$ side is hidden behind the bar material and suffers higher extinction.

Moreover, the dust extinction is highest in a curved structure just W of the nucleus, which seems to be connected to the dust lane in the bar through an higher extinction ridge, visible at the bottom part of the map and around clump B (see also Figs. 3c, and 1 panel b, where the dust lane is visible as a dip in the continuum shown in green). This dusty structure appears to be $\sim 7^{\prime \prime} \times 1.5^{\prime \prime}$ in size, corresponding to $580 \times 125 \mathrm{pc}$, and shows the highest reddening in the central region of the galaxy, with $A_{V}>3$ and a maximum of $A_{V}=4.8$. The structure is also evident in Fig. 1, as a dip in the shorter wavelength emission line map, [OIII] $\lambda 5007$ shown in red, as well in Fig. 3c, where the ratio between the continuum flux in the 8000-8500 $\AA$ spectral region and the 5100-5500 $\AA$ spectral region is shown. While the star-forming clumps and the nucleus are bluer as a result of 

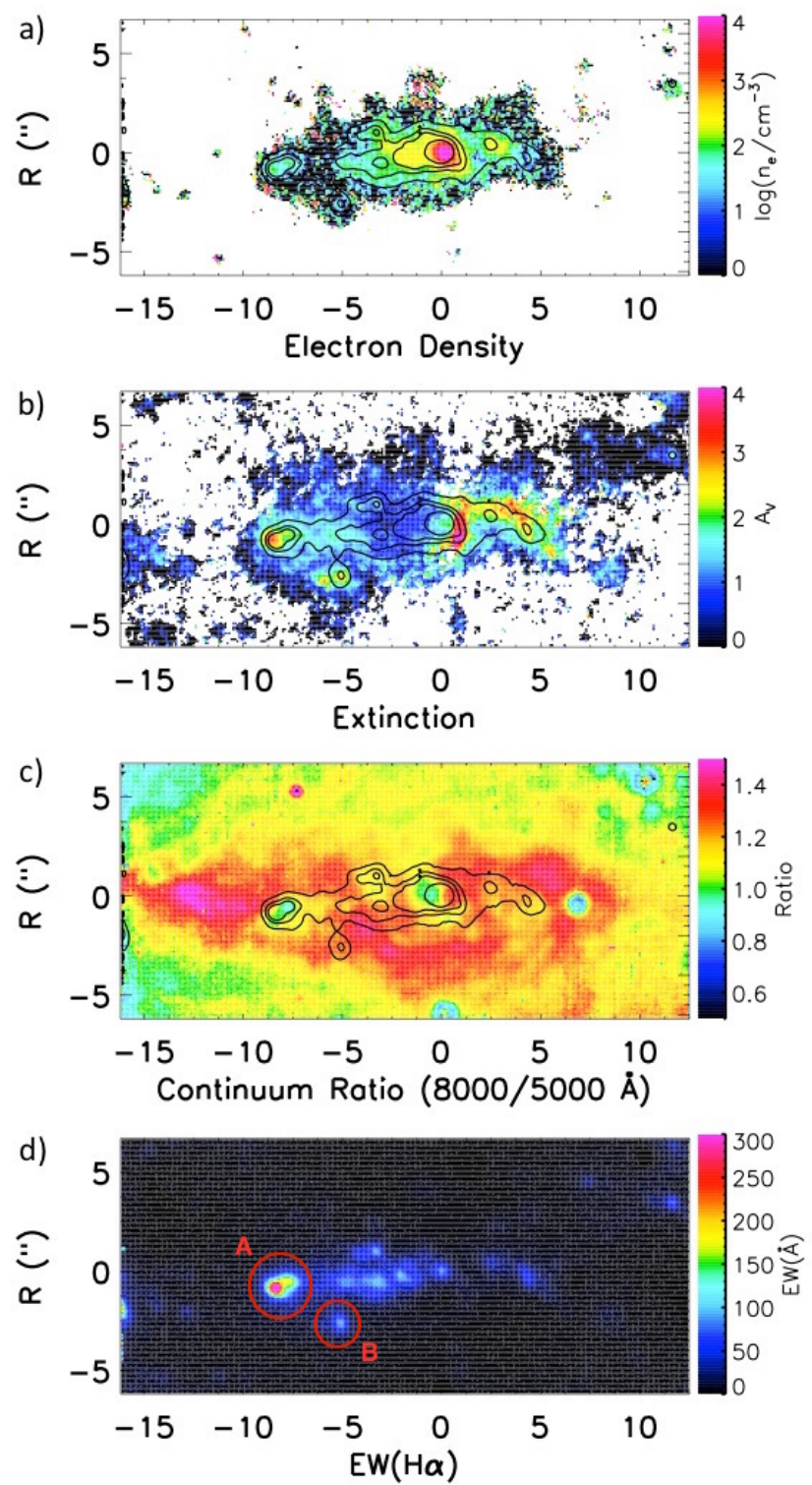

Fig. 3. Electron density (panel a)) and extinction map (panel b)), as derived from the $[\mathrm{SII}] \lambda 6716 /[\mathrm{SII}] \lambda 6730$ and $\mathrm{H} \alpha / \mathrm{H} \beta$ line ratios (see text) with $\mathrm{H} \alpha$ contours overplotted. The star-forming clump has higher density and extinction than its surroundings. A dusty structure with very high extinction is detected just west of the nucleus and $\mathrm{H} \alpha$ peak, corresponding to the tip of the bar driven dust lane. Both maps show the spaxels with $S / N>5$. The ratio between the continuum flux in the 8000-8500 ̊ spectral region and the 5100-5500 Å spectral region, with $\mathrm{H} \alpha$ contours overplotted, is shown in panel c). While the star-forming clumps and the nucleus are bluer due to the intrinsic shape of the continuum, the higher extinction regions show redder colors, tracing the dust distribution in the regions even where the $\mathrm{S} / \mathrm{N}$ of the emission lines is lower. The dust lanes to the $\mathrm{W}$ and $\mathrm{E}$ of the nucleus, an extinction ridge to the $\mathrm{S}$ connecting the dust lane with the dust structure surrounding the nucleus are clearly visible. Panel d) shows the rest-frame $\mathrm{H} \alpha$ Equivalent Width map. The star-forming clump A, marked with a circle as well as clump B, shows the highest $E W(\mathrm{H} \alpha)=350 \AA$ in the field of view, suggesting an age of $\sim 10^{7} \mathrm{yrs}$ (see text for details).

the intrinsic shape of the continuum, the regions with higher extinction have redder colors, tracing the dust distribution in the regions even where the $\mathrm{S} / \mathrm{N}$ of the emission lines is lower. In this continuum ratio map, the dust lanes to the $\mathrm{W}$ and $\mathrm{E}$ of the nucleus and the higher extinction ridge to the $\mathrm{S}$, connecting the dust lane with the dust structure surrounding the nucleus, are clearly visible. It has been suggested that this dusty region represents the tip of the bar in the nuclear region, where the flowing material forms a disky structure elongated perpendicularly to the bar itself (e.g., Morris et al. 1985). This is actually predicted by dynamical models, where the gas is expected to settle into a disk with rotation axis along the bar (see, e.g., Durisen et al. 1983). It is possibly also responsible for the collimation of the ionizing photons along the bar (Menezes et al. 2015), generating the ionization bicone seen in [OIII] line emission. The alignment between the inner bar and the EELR is therefore actually expected, with the ionizing continuum that escapes in this case in a cone slightly tilted with respect to the bar so that the gas is mostly illuminated in front of the bar to the $\mathrm{E}$ and behind the bar to the W. This high extinction region is also dominated by shock ionization, as discussed in Sect. 3.

\section{Resolved BPT diagrams}

We use the measured emission line fluxes in each spaxel to compute excitation maps of the galaxy region within the MUSE field of view. We consider both the classical Baldwin et al. 1981 BPT diagram using the [OIII] $\lambda 5007 / \mathrm{H} \beta$ versus $[\mathrm{NII}] \lambda 6584 / \mathrm{H} \alpha$ line ratios (N-BPT in the following), as well as the alternative versions using [SII] $\lambda 6716,30$ (S-BPT) or [OI] 76300 instead of [NII] $\lambda 6584$ (O-BPT; see, e.g., Kewley et al. 2006; Lamareille 2010). In these diagrams, galaxies dominated by AGN (Seyfert-type), low-ionization nuclear emission line regions (LINERs), shocks, and star formation ionization populate different regions. For the N-BPT diagram, we also plot the location of points for two prototypical galaxies, the shockexcited region of NGC 1482 and the AGN-excited emission of NGC 1365, from Sharp \& Bland-Hawthorn 2010. The bisector between these two fiducial traces is used to discriminate between AGN-excited and shock-excited gas in the N-BPT diagram. In the right panels of Fig. 4, we show the maps where different regions have been color coded according to their line excitation as derived from the corresponding BPT diagram on the left. The HII regions are marked in blue, AGN-dominated regions are in green, and LINER-like or shock-excited regions are marked in red, while composite regions in the N-BPT are shown in magenta (see Kewley et al. 2006; Kauffmann et al. 2003). In all BPT maps the nuclear region, the EELR and the ionization cone are dominated by AGN ionization. Shock-excited gas is prevalent in the surrounding region and at the location of the dusty structure surrounding the nucleus, while star formation is dominant at the edges of the MUSE field of view, in a circumnuclear star-forming ring. The only notable exception to this schematic picture are the blue, $\mathrm{H} \alpha$ bright regions already prominent in Fig. 1. Although they are located at the tip of the central AGNdominated region and along the ionization cone, they are classified as star formation dominated by all three BPT diagrams (clump A), or by two out of three (clump B, see Figs. 4 and 7). Interestingly, comparing the location of clump A and B in Fig. 1, they also appear to be located on the ridge of the dust lane in the bar.

Clump $\mathrm{A}$ is also the region with the highest $\mathrm{H} \alpha \mathrm{EW}$ in the field of view, $E W(\mathrm{H} \alpha)=350 \AA$ rest frame (see Fig. 3d). The other star-forming regions, mostly located in a star-forming ring near the edge of the MUSE field of view do not exceed $E W(\mathrm{H} \alpha)=250 \AA$. The high EW in Clump A suggests a young age of its stellar population. Using the Starburst99 models (Leitherer et al. 1999), assuming a solar metallicity and a Salpeter IMF, we obtain an estimate of the age of the cluster of 

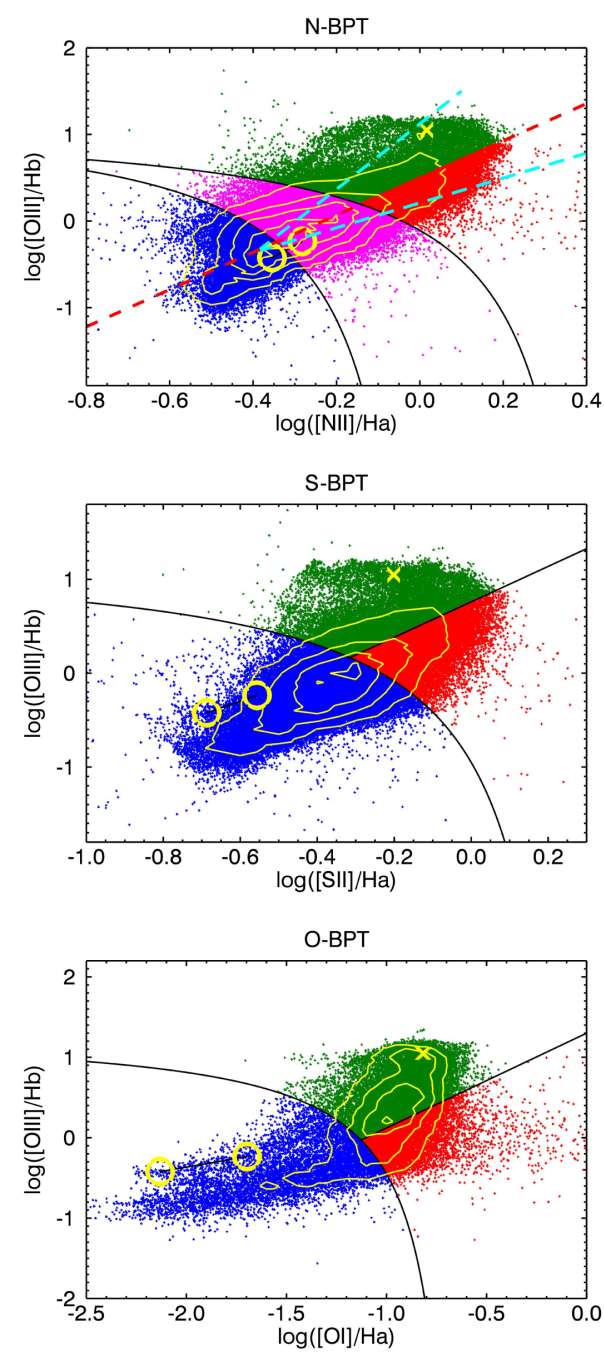

N-BPT map

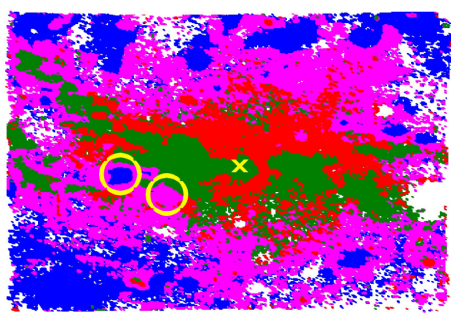

S-BPT map

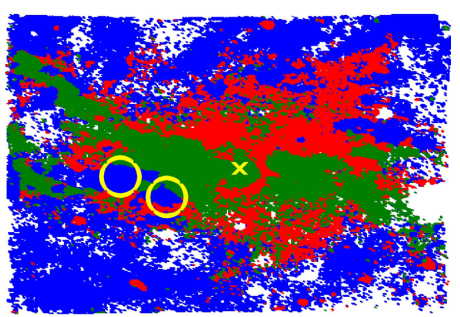

O-BPT map

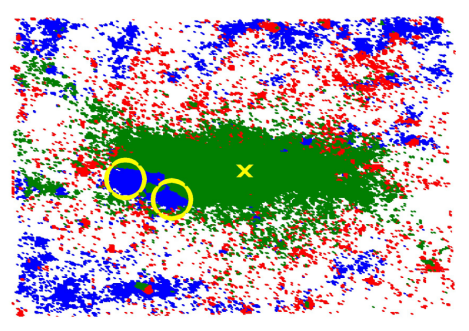

Fig. 4. Resolved BPT diagrams for the nuclear region of NGC 5643. The N-BPT ([NII] $] 6584 / \mathrm{H} \alpha$ vs. [OIII] $] 25007 / \mathrm{H} \beta, \quad$ upper panels), S-BPT ([SII] $\lambda 6716,30 / \mathrm{H} \alpha$ vs. $[\mathrm{OIII}] \lambda 5007 / \mathrm{H} \beta$, central panels) and O-BPT $[\mathrm{OI}] \lambda 6300 / \mathrm{H} \alpha$ vs. $[\mathrm{OIII}] \lambda 5007 / \mathrm{H} \beta$, lower panels) diagrams for each spaxel with $S / N>3$ in each line are shown on the left, along with contours showing the density of the points on the diagram. The location of the $\mathrm{H} \alpha$ bright clumps $\mathrm{A}$ and $\mathrm{B}$, as derived from an integrated spectrum extracted on a region of $1.6 \times 1.6^{\prime \prime}$, is shown with a yellow circle, while a cross marks the location of the nucleus. The dashed cyan lines in the N-BPT mark the location of points for the shock-excited region of NGC 1482 and for the AGN-excited emission of NGC 1365 from Sharp \& Bland-Hawthorn 2010. The bisector between the two, marked with a dashed red line, divide AGN-dominated by shock-dominated regions. A map marking each spaxel with the color corresponding to the dominant excitation at its location is shown on the right, again for spaxels with $S / N>3$. The star-forming regions are marked in blue, the Seyfert-type ionization is shown in green, LINER- or shock-dominated regions are shown in red, and intermediate regions are shown in magenta in the N-BPT. Star-forming regions are prevalent in the outer part of the MUSE field of view, AGN ionization is dominating in the core and in the ionization cone, while shock excitated gas is prevalent around the EELR and the cone. The nucleus is marked with a cross, and the $\mathrm{H} \alpha$ bright clups $\mathrm{A}$ and $\mathrm{B}$ are marked with a yellow circle. Clump A is dominated by star formation in all the three diagrams, while Clump B is classified as intermediate by the N-BPT. $t_{\text {cluster }}=5.5 \times 10^{6} \mathrm{yr}$ for an instantaneous burst, and $t_{\text {cluster }}=$ $8.7 \times 10^{7} \mathrm{yr}$ for a continuous burst of star formation. These values are considered as upper limits, as the measured continuum under the line is integrated over a line of sight crossing of the whole galaxy, not only of the star-forming region, and therefore the measured $\mathrm{EW}(\mathrm{H} \alpha)$ is considered a lower limit to the true value. From the measured $\mathrm{H} \alpha$ flux extracted in a region of $1.6^{\prime \prime} \times 1.6^{\prime \prime}$ around the clump, assuming the star formation rate calibration of Kennicutt \& Evans 2012 and correcting for dust extinction using the Balmer decrement $\mathrm{H} \alpha / \mathrm{H} \beta$ assuming a Calzetti 2000 extinction law, we derive a star formation rate in the clump of $S F R_{\text {(clump) }}=0.03 M_{\odot} / \mathrm{yr}$ (corresponding to $\Sigma_{\mathrm{SFR}}=1.7 M_{\odot} \mathrm{yr}^{-1} \mathrm{kpc}^{-2}$ ). Given that clump B is classified as intermediate by the N-BPT, we do not measure a SFR from $\mathrm{H} \alpha$, as it may be contaminated by AGN ionization. The spectrum of the clumps is characterized by an almost featureless continuum as expected from the cluster age estimated from the $E W(\mathrm{H} \alpha)$. One might analyze the Balmer lines in absorption to derive a more reliable stellar age, but, with such a strong infilling emission $(\mathrm{EW}(\mathrm{H} \alpha)>300 \AA)$, to reliably measure the Balmer absorptions one would require a much higher $\mathrm{S} / \mathrm{N}$ in the continuum than is actually available. Actually, the emission is so strong that it completely obliterates any absorption in these clumps. We discuss in the next section why these particular locations are so special, and what has probably triggered star formation in these clumps.

\section{Outflowing gas and positive feedback}

The presence of high velocity gas in the EELR region is revealed by the ionized gas dynamics traced by our MUSE observations. The nuclear spectrum shown in Fig. 2 presents an asymmetric profile of the [OIII] lines. The [OIII] lines are an ideal tracer of extended outflowing ionized gas, as they cannot be produced in the high-density, sub-parsec scales typical of AGN broad-line regions (BLR), and have been widely used in the literature to study the winds properties (e.g., Villar-Martín et al. 2011; Zhang et al. 2011; Brusa et al. 2015). A similar, asymmetric profile is observed for other forbidden lines, as the [NII] and [SII] doublets.

We fit the $\mathrm{H} \beta$, [OIII] $\lambda 4959$ and [OIII] $\lambda 5007$ nuclear spectrum of NGC 5643 (in a region of $1.6^{\prime \prime} \times 1.6^{\prime \prime}$ ) with a double Gaussian for each line, a systemic component and an outflowing component (see Fig. 5). All the lines are fitted simultaneously, fixing the wavelengths ratios and [OIII] flux ratios according to theoretical values. The systemic components have a $F W H M_{\text {sys }}=286 \pm 6 \mathrm{~km} \mathrm{~s}^{-1}$, while the outflowing components have a centroid shifted by $v_{0, \text { out }}=-200 \pm 15 \mathrm{~km} \mathrm{~s}^{-1}$ and a $F W H M_{\text {out }}=590 \pm 20 \mathrm{~km} \mathrm{~s}^{-1}$. The projected velocity at the 10 th percentile of the overall emission-line profile of the best-fitting model is $v_{10}=-430 \mathrm{~km} \mathrm{~s}^{-1}$. These high velocities confirm the presence of outflowing gas from the nuclear region.

Thanks to our IFU data, we are able to spatially map this high velocity gas: the upper panel of Fig. 6 shows the contours 


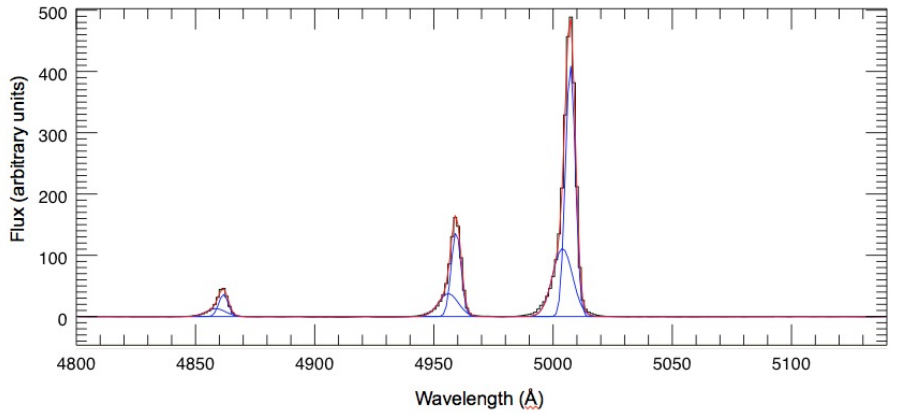

Fig. 5. Two-component Gaussian fitting of the $1.6^{\prime \prime} \times 1.6^{\prime \prime}$ rest-frame nuclear spectrum of NGC 5643 for the $\mathrm{H} \beta,[\mathrm{OIII}] \lambda 4959$ and [OIII] $\lambda 5007$ emission lines. The [OIII] $\lambda 5007$ lines in the nucleus shows an asymmetric blue wing, reproduced with blueshifted Gaussian components. The systemic components have a $F W H M_{\text {sys }}=286 \pm 6 \mathrm{~km} \mathrm{~s}^{-1}$, while the outflowing components have a centroid shifted by $v_{0, \text { out }}=-200 \mathrm{~km} \mathrm{~s}^{-1}$ and a $F W H M_{\text {out }}=590 \mathrm{~km} \mathrm{~s}^{-1}$. The projected velocity at the 10th percentile of the overall emission-line profile is $v_{10}=-430 \mathrm{~km} \mathrm{~s}^{-1}$, confirming the presence of outflowing, high velocity gas from the nuclear region.

of high velocity [OIII] $\lambda 5007$ emitting gas, derived by integrating the $[\mathrm{OIII}] \lambda 5007$ emission in the spectral channels with $v<$ $-400 \mathrm{~km} \mathrm{~s}^{-1}$ with respect to the bulk velocity of the systemic component. The contours are superimposed on the $\mathrm{H} \alpha$ map.

NGC 5643 is also known to host a double-sided diffuse radio jet, aligned with the bar, which extends by $\sim 15^{\prime \prime}=1.2 \mathrm{kpc}$ in both the $\mathrm{E}$ and $\mathrm{W}$ direction. The radio jet is observed by Morris et al. 1985 at 6 and $20 \mathrm{~cm}$, and by Leipski et al. 2006 at $3.5 \mathrm{~cm}$. The higher resolution $\left(1.7^{\prime \prime} \times 1.7^{\prime \prime}\right.$ beam $) 3.5 \mathrm{~cm}$ VLA contours are shown in Fig. 6, central panel, superimposed on the $\mathrm{H} \alpha$ emission line map from our MUSE data. The radio emission morphology is similar to the EELR, although the $\mathrm{H} \alpha$ emission is suppressed in the $\mathrm{W}$ direction due to the higher extinction. Given the presence of a low-luminosity radio jet, the high velocity ionized gas could be accelerated by a sort of "snowplow" effect, with the high velocity shocks induced by the outflowing plasma heating and compressing the external gas (see, e.g., Axon et al. 1998; Capetti et al. 1999).

An alternative mechanism to explain the detected radio emission could be the interaction between an accretion disk wind and the surrounding ISM (see, e.g., Faucher-Giguère \& Quataert 2012; Zakamska \& Greene 2014; Nims et al. 2015; Harrison et al. 2015). In fact, the radio luminosity of NGC 5643 is $v L_{v}[8.4 \mathrm{GHz}]=0.55 \times 10^{21} \mathrm{erg} \mathrm{s}^{-1}$ (Leipski et al. 2006), requiring a kinetic energy of the outflow of $L_{\text {wind }} \sim 2 \times 10^{39} \mathrm{erg} \mathrm{s}^{-1}$ if the efficiency of conversion in AGN driven winds is comparable to that in supernova driven winds. Thus the kinetic luminosity of the wind would be $\sim 1 \%$ of the bolometric luminosity of NGC 5643, making this scenario viable. In any case, the radio emission is consistent with being produced by a low-luminosity radio jet or quasar wind that are interacting with the ISM in the host galaxy.

We also show the X-ray images obtained filtering the Chandra data in the $0.3-0.5 \mathrm{keV}$ and $0.5-8 \mathrm{keV}$ energy bands in Fig. 6, bottom panel, where the high energy data are shown in blue and the lower energy data are shown in green. The high energy X-ray emission is concentrated in the central region, mostly in the unresolved central point source, with a minor fraction spread over a few arcsec around the center. This is analogous to what has been observed in other similar sources (e.g., NGC 1365, Wang et al. 2009; NGC 4151, Wang et al. 2010). The unresolved emission is due to the direct emission of the central
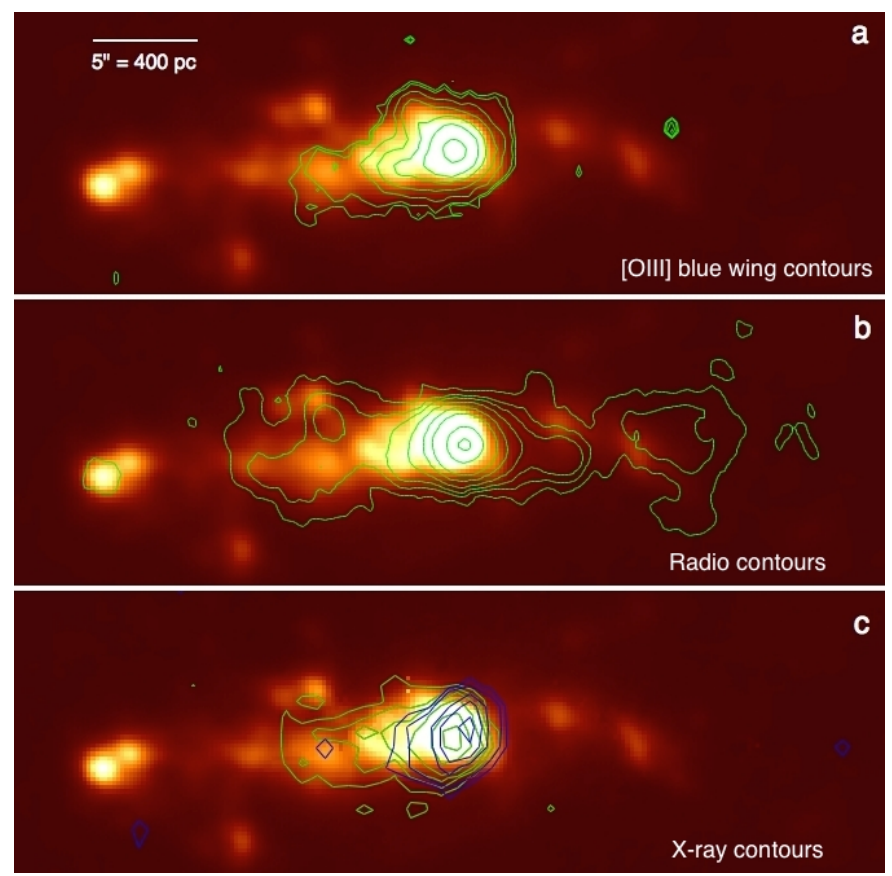

Fig. 6. Outflowing gas in the core of NGC 5643. Panel a) $\mathrm{H} \alpha$ map of the central region, with superimposed the contours of the flux in the blue wing $\left(v<-400 \mathrm{~km} \mathrm{~s}^{-1}\right)$ of the [OIII] $\lambda 4959$ emission line, tracing the high velocity outflowing ionized gas. Panel b) $\mathrm{H} \alpha$ map with superimposed the $8.4 \mathrm{GHz}$ radio contours from the VLA observations presented in Leipski et al. 2006 . The diffuse radio jet is coincident with the location of the blue [OIII] $\lambda 4959$ wing. Panel c) $\mathrm{H} \alpha$ map with superimposed Chandra X-ray contours, $0.3-0.5 \mathrm{keV}$ shown in green, and $0.5-8 \mathrm{keV}$ shown in blue. While the high energy data are only located in the nuclear region, the lower energy channels show extended emission compatible with an outflowing jet. Again, the location of the jet traced by $\mathrm{X}$-rays corresponds to the ionized gas and radio emitting gas, and points toward the star-forming blue clump, suggesting a possible connection between the star-forming episode and the outflowing material.

AGN (including the primary emission and the reflected component by the circumnuclear gas on a parsec scale). The spatially resolved hard emission is expected to be due to a combination of reflection of the primary AGN emission by farther circumnuclear gas, at a distance of a few hundred pc, and the contribution of galactic X-ray binaries. Disentangling these components would require a higher $\mathrm{S} / \mathrm{N}$ observation than available here, and will become possible with a forthcoming long Chandra observation scheduled in 2015. The soft emission is instead dominated by the extended, spatially resolved component, and is due to the emission of hot gas (typical temperatures $k T \sim 0.2-1 \mathrm{keV}$ ) either shock heated, or photoionized by the central AGN. In particular, the spatial coincidence of the soft X-ray emission and the [OIII] emission points to a common origin of these two components, both due to photoionization by the central AGN of a twophase gas, with a denser phase associated with the [OIII] emission and the less dense, more photoionized one responsible for the X-ray emission (e.g., Bianchi et al. 2006; Balmaverde et al. 2012).

The outflow direction as detected by radio, X-ray, and ionized gas dynamics is pointing toward the location of the blue, $\mathrm{H} \alpha$ bright clumps, suggesting a mutual connection between the star formation in the clumps and the outflowing gas. In fact, we have seen in Sect. 2.2 that star-forming clumps A and B are not only on the path of the material outflowing from the nucleus, but they are also located at the receding edge of the dust lane 


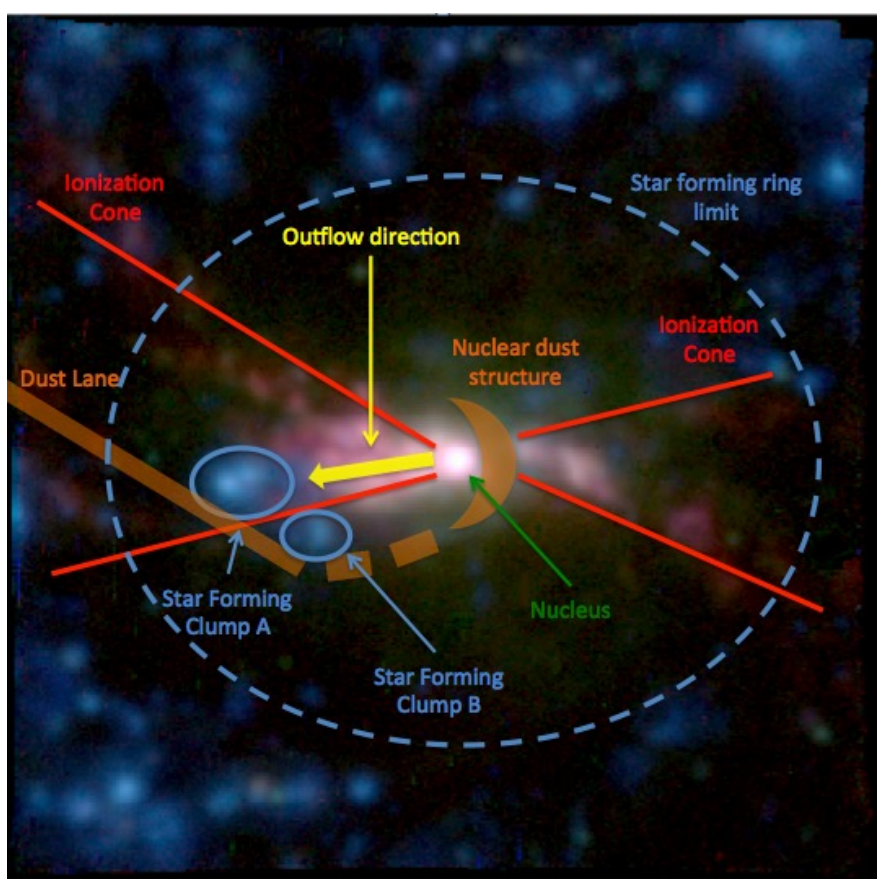

Fig. 7. Schematic view of the main structures revealed by MUSE in the central region of NGC 5643, drawn over the three-color image from Fig. 1, panel c). The ionization cone borders are highlighted with red lines, the dust lane and nuclear dust structure location in brown. The two star-forming clumps A and B are marked with blue circles, and the dashed blue ellipse, corresponding to a circle with the same inclination of the galaxy, shows the inner radius of the star-forming ring around the nuclear region of the galaxy.

in the bar. Bars are in fact preceded by dust lanes in their motion, which have been recognized as related to shocks in the gas flow, and correspond to the regions of highest gas density (e.g., Athanassoula 1992). Despite the high density, the gas flow is accompanied by a very strong shear that inhibits star formation. These shocks are usually found in the leading edge of the bar, roughly parallel to its major axis. Instead, H II regions and star formation are preferentially offset from the dust lane, toward the leading side, as well as at the end of the bar major axis, where secondary gas density enhancements are seen both in simulations and observationally (e.g., Sheth et al. 2002). As clumps A and $\mathrm{B}$ are located on the trailing edge of the dust lane, they are not at the expected position for $\mathrm{H}$ II regions surrounding a bar, reinforcing the hypothesis of a mutual connection with the nuclear outflow. In this scenario, the outflow has been compressing the gas in the dust lane at the intersection point, triggering star formation ("positive feedback").

\section{Conclusions}

We have presented MUSE integral field data of NGC 5643, a local barred Seyfert 2 galaxy at a distance of $\sim 17.3 \mathrm{Mpc}$. The large wavelength coverage allows us to study the main optical emission lines in the spectra and map the ionization and dynamics of the gas.

We detected a double-sided ionization cone due to AGN radiation, almost parallel to the galaxy bar. The cone may be collimated by a dusty structure of $580 \times 125 \mathrm{pc}$ around the nucleus, connected with the dust lane in the bar and dominated by shock ionization. At the center of the cone an outflow is revealed by the high velocity, [OIII] emitting gas, as well as in radio and
$\mathrm{X}$-ray data. The ionized gas is moving away from the nucleus at projected velocities up to $v_{10}=-432 \mathrm{~km} \mathrm{~s}^{-1}$.

Two $\mathrm{H} \alpha$ bright, star formation dominated clumps, located at the receding edge of the dust lane in the bar, where the gas density is highest, are located at the location where the fast outflowing gas from the central AGN encounter the dense material on the bar, strongly suggesting a relation between the two phenomena. The clumps are the youngest in the MUSE field of view, as estimated from their $\mathrm{H} \alpha(\mathrm{EW})$, and are the only star formation dominated regions closer to the nucleus $(\sim 1.2 \mathrm{kpc})$ than the starforming ring located at the edge of the field of view $(\sim 2.3 \mathrm{kpc}$, see Fig. 7). We propose that the star formation in the clumps ( $S F R=0.03 M_{\odot} / \mathrm{yr}$ for clump A) is due to positive feedback of the AGN outflow, which is compressing the gas at the dust lane edge inducing star formation (see Fig. 7).

The presented data suggest that positive feedback may also be present at low AGN luminosity and energetic outflow. The upcoming observations in the framework of the MAGNUM survey will allow us to assess if positive feedback represents an important ingredient in the complex black hole-host galaxy coevolution also in the local Universe.

Acknowledgements. MUSE data were obtained from observations made with the ESO Telescopes at the Paranal Observatories. We are grateful to the ESO staff for their work and support. We are grateful to C. Leipski for providing us the VLA radio data of NGC 5643. G.C., A.M., and S.Z. acknowledge support from grant PRIN-INAF 2011 "Black hole growth and AGN feedback through the cosmic time" and from grant PRIN-MIUR 2010-2011 "The dark Universe and the cosmic evolution of baryons: from current surveys to Euclid". S.Z. and A.G. have been supported by the EU Marie Curie Integration Grant "SteMaGE" No. PCIG12-GA-2012-326466 (Call Identifier: FP7-PEOPLE-2012 CIG). A.G. acknowledges support from the European Union FP7/2007-2013 under grant agreement No. 267251 (AstroFIt). M.B. acknowledges support from the FP7 Career Integration Grant "eEASy” (CIG 321913).

\section{References}

Athanassoula, E. 1992, MNRAS, 259, 345

Axon, D. J., Marconi, A., Capetti, A., et al. 1998, ApJ, 496, L75

Bacon, R., Accardo, M., Adjali, L., et al. 2010, Proc. SPIE, 7735, 773508

Baldwin, J. A., Phillips, M. M., \& Terlevich, R. 1981, PASP, 93, 5

Balmaverde, B., Capetti, A., Grandi, P., et al. 2012, A\&A, 545, A143

Bruzual, G., \& Charlot, S. 2003, MNRAS, 344, 1000

Brusa, M., Bongiorno, A., Cresci, G., et al. 2015, MNRAS, 446, 2394

Bianchi, S., Guainazzi, M., \& Chiaberge, M. 2006, A\&A, 448, 499

Calzetti, D., Armus, L., Bohlin, R. C., et al. 2000, ApJ, 533, 682

Capetti, A., Axon, D. J., Macchetto, F. D., Marconi, A., \& Winge, C. 1999, ApJ, 516, 187

Cappellari, M., \& Emsellem, E. 2004, PASP, 116, 138

Cresci, G., Mainieri, V., Brusa, M., et al. 2015, ApJ, 799, 82

Crockett, R. M., Shabala, S. S., Kaviraj, S., et al. 2012, MNRAS, 421, 1603

Croft, S., van Breugel, W., de Vries, W., et al. 2006, ApJ, 647, 1040

de Vaucouleurs, G., de Vaucouleurs, A., \& Corwin, J. R. 1976, Second reference catalogue of bright galaxies (Austin: University of Texas Press)

Durisen, R. H., Tohline, J. E., Burns, J. A., \& Dobrovolskis, A. R. 1983, ApJ, 264,392

Elbaz, D., Jahnke, K., Pantin, E., Le Borgne, D., \& Letawe, G. 2009, A\&A, 507, 1359

Fabian, A. C. 2012, ARA\&A, 50, 455

Faucher-Giguère, C.-A., \& Quataert, E. 2012, MNRAS, 425, 605

Feain, I. J., Papadopoulos, P. P., Ekers, R. D., \& Middelberg, E. 2007, ApJ, 662, 872

Fischer, T. C., Crenshaw, D. M., Kraemer, S. B., \& Schmitt, H. R. 2013, ApJS, 209, 1

Fruscione, A., McDowell, J. C., Allen, G. E., et al. 2006, Proc. SPIE, 6270, $62701 \mathrm{~V}$

Garmire, G. P., Bautz, M. W., Ford, P. G., Nousek, J. A., \& Ricker, G. R., Jr. 2003, Proc. SPIE, 4851, 28

Harrison, C. M., Thomson, A. P., Alexander, D. M., et al. 2015, ApJ, 800, 45

Hinshaw, G., Larson, D., Komatsu, E., et al. 2013, ApJS, 208, 19

Ho, L. C., Li, Z.-Y., Barth, A. J., Seigar, M. S., \& Peng, C. Y. 2011, ApJS, 197, 21 
G. Cresci et al.: Positive feedback in the nuclear region of NGC 5643 suggested by MUSE

Kauffmann, G., Heckman, T. M., Tremonti, C., et al. 2003, MNRAS, 346, 1055 Kennicutt, R. C., \& Evans, N. J. 2012, ARA\&A, 50, 531

Kewley, L. J., Groves, B., Kauffmann, G., \& Heckman, T. 2006, MNRAS, 372, 961

Klamer, I. J., Ekers, R. D., Sadler, E. M., \& Hunstead, R. W. 2004, ApJ, 612, L97

Koribalski, B. S., Staveley-Smith, L., Kilborn, V. A., et al. 2004, AJ, 128, 16

Imanishi, M., Ichikawa, K., Takeuchi, T., et al. 2011, PASJ, 63, 447

Ishibashi, W., \& Fabian, A. C. 2012, MNRAS, 427, 2998

Ishibashi, W., \& Fabian, A. C. 2014, MNRAS, 441, 1474

Lamareille, F. 2010, A\&A, 509, A53

Leipski, C., Falcke, H., Bennert, N., Hüttemeister, S. 2006, A\&A, 455, 161

Leitherer, C., Schaerer, D., Goldader, J. D., et al. 1999, ApJS, 123, 3

Menezes, R. B., da Silva, P., Ricci, T. V., et al. 2015, MNRAS, 450, 369

Morris, S., Ward, M., Whittle, M., Wilson, A. S., \& Taylor, K. 1985, MNRAS, 216,193

Mullaney, J. R., Daddi, E., Béthermin, M., et al. 2012, ApJ, 753, L30

Nims, J., Quataert, E., \& Faucher-Giguère, C.-A. 2015, MNRAS, 447, 3612

Osterbrock, D. E., \& Ferland, G. J. 2006, Astrophysics of gaseous nebulae and active galactic nuclei, D. E. Osterbrock, \& G. J. Ferland, 2nd. eds. (Sausalito, CA: University Science Books)

Rodríguez Zaurín, J., Holt, J., Tadhunter, C. N., \& González Delgado, R. M. 2007, MNRAS, 375, 1133
Salomé, Q., Salomé, P. \& Combes, F. 2015, A\& A, 574, A34

Sarzi, M., Falcón-Barroso, J., Davies, R. L., et al. 2006, MNRAS, 366, 1151

Schmitt, H. R., Storchi-Bergmann, T., \& Baldwin, J. A. 1994, ApJ, 423, 237

Sharp, R. G., \& Bland-Hawthorn, J. 2010, ApJ, 711, 818

Sheth, K., Vogel, S. N., Regan, M. W., et al. 2002, AJ, 124, 2581

Silk, J. 2013, ApJ, 772, 112

Silk, J., \& Norman, C. 2009, ApJ, 700, 262

Silverman, J. D., Kovač, K., Knobel, C., et al. 2009, ApJ, 695, 171

Simpson, C., Wilson, A. S., Bower, G., et al. 1997, ApJ, 474, 121

Villar-Martín, M., Humphrey, A., Delgado, R. G., Colina, L., \& Arribas, S. 2011, MNRAS, 418, 2032

Wang, J., Fabbiano, G., Elvis, M., et al. 2009, ApJ, 694, 718

Wang, J., Fabbiano, G., Risaliti, G., et al. 2010, ApJ, 719, L208

Wang, J., Fabbiano, G., Elvis, M., et al. 2011, ApJ, 742, 23

Weilbacher, P. M., Streicher, O., Urrutia, T., et al. 2014, Astronomical Data Analysis Software and Systems XXIII, 485, 451

Zakamska, N. L., \& Greene, J. E. 2014, MNRAS, 442, 784

Zhang, K., Dong, X.-B., Wang, T.-G., \& Gaskell, C. M. 2011, ApJ, 737, 71

Zibetti, S. 2009, Arxiv e-prints [arXiv:0911.4956]

Zibetti, S., Charlot, S., \& Rix, H.-W. 2009, MNRAS, 400, 1181

Zinn, P.-C., Middelberg, E., Norris, R. P., \& Dettmar, R.-J. 2013, ApJ, 774, 66 Zubovas, K., Nayakshin, S., King, A., \& Wilkinson, M. 2013, MNRAS, 433 , 3079

Zubovas, K. \& King, A. R. 2014, MNRAS, 439, 400 